\title{
THE ROLE OF HYALURONAN IN THE TREATMENT OF EPIDERMAL ATROPHY
}

Lenka Machková', Iva Dolečková'

' Contipro a.s., Dolní Dobrouč, Czech Republic

\section{INTRODUCTION}

Senile skin atrophy is a common side effect of intrinsic aging and UV exposition and it affects both dermal and epidermal layers of the skin. While dermal atrophy is mostly associated with the loss of connective tissue, epidermal atrophy manifests as overall thinning of epidermis, reduction of keratinocyte size and flattening of the dermoepidermal junction. Compared to senile atrophy, steroid skin atrophy frequently arises during glucocorticoid therapy and causes serious skin damages, particularly when applied to fragile skin.

\section{MATERIALS AND METHODS}

In this study, we investigated the ability of $0,1 \%$ high (1500 - 1800 kDa, HMW-SH), low (320 kDa, LMW$\mathrm{SH})$ and very low (15 kDa, VLMW$\mathrm{SH})$ molecular weight sodium hyaluronate $(\mathrm{SH})$ to reverse the general signs of the skin atrophy (decreased cell viability, lower total protein level) and modulate the specific signs of epidermal atrophy (expression of matrix metalloproteinases) in the in vitro model of dexamethasone-treated HaCaT keratinocytes. We also performed in vivo study to evaluate the effect of VLMW-SH and LMW-SH topical application on thickness of living epidermis using the method of in vivo reflectance confocal microscopy.

\section{RESULTS}

The anti-atrophic potential of sodium hyaluronate $(\mathrm{SH})$ has been studied previously and it was demonstrated on a group of patients affected by steroid atrophy'.

Our results show that VLMW-SH significantly reverses all the signs of dexamethasone-induced atrophy in $\mathrm{HaCaT}$ keratinocytes investigated in this study and increases the average epidermal thickness of aged skin in vivo. HMW-SH demonstrated less pronounced effect and LMW-SH showed no significant anti-atrophic abilities neither in vitro nor in vivo.

\section{CONCLUSION}

In conclusion, we confirmed the importance of hyaluronan in senile and steroid-induced skin atrophy. However, therapeutic potentials of HMW, LMW and VLMW-SH show significant differences and should be investigated in detail. These results support the beneficial effects of $\mathrm{SH}$ and the possibility of its application in the therapy of atrophic epidermal skin.
INVITROANTI-ATROPHICEFFECTOFHMW, LMW and VLMW SODIUM HYALURONATE

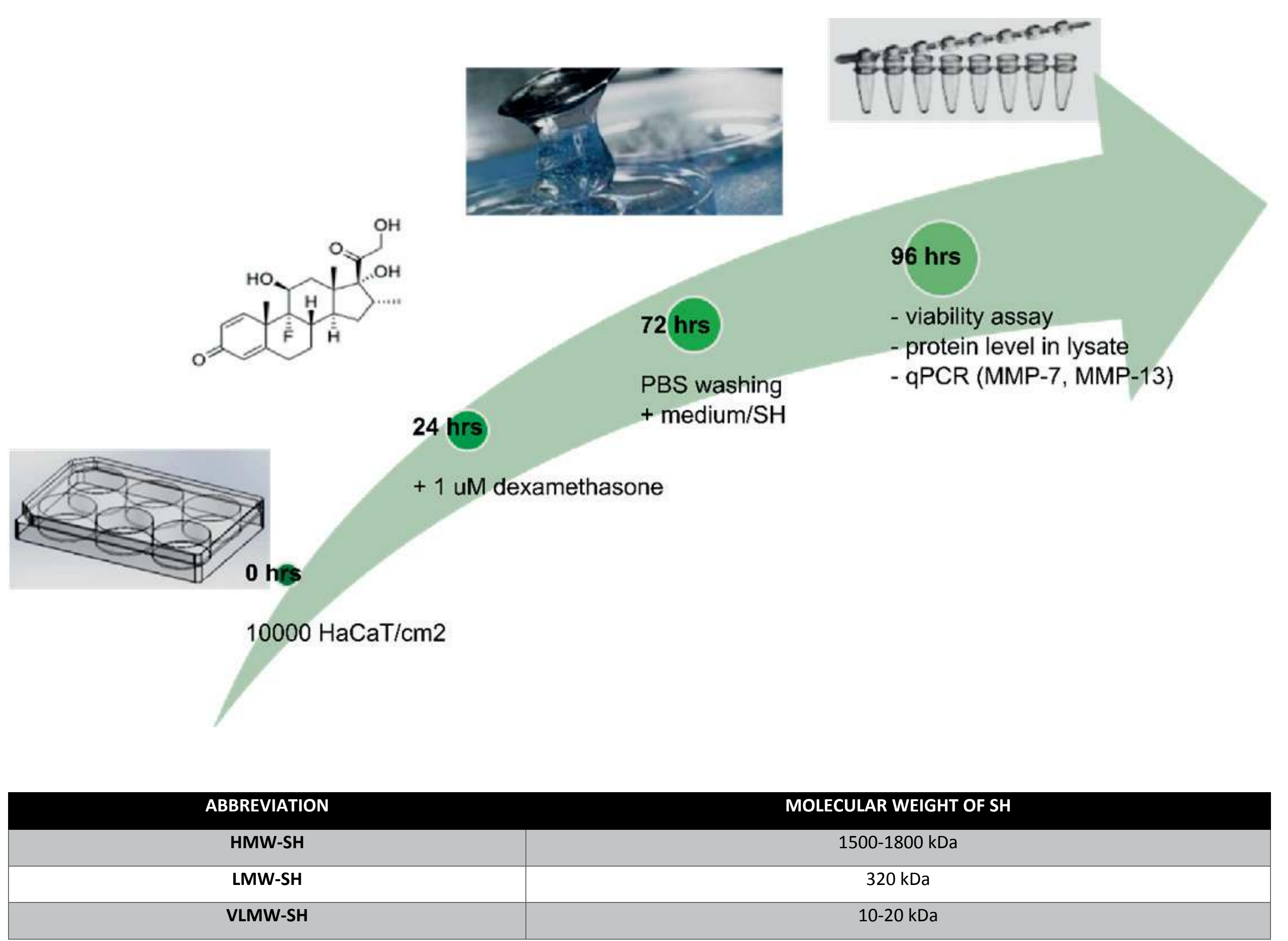

Figure 1. In vitro model of dexamethasone-induced atrophy. $\mathrm{HaCaT}$ keratinocytes were treated with $1 \mu \mathrm{M}$ dexamethasone for $72 \mathrm{hrs}$, then the cells were washed, cultivated in fresh medium with/without SH. Sodium hyaluronate used in this study was produced using a non-haematolytic microbial strain Streptococcus equi. After $24 \mathrm{hrs}$, the markers of atrophy strain Streptococcus investigated.

Table 1. Markers of atrophy studied in dexamethasonetreated $\mathrm{HaCaT}$ keratinocytes.
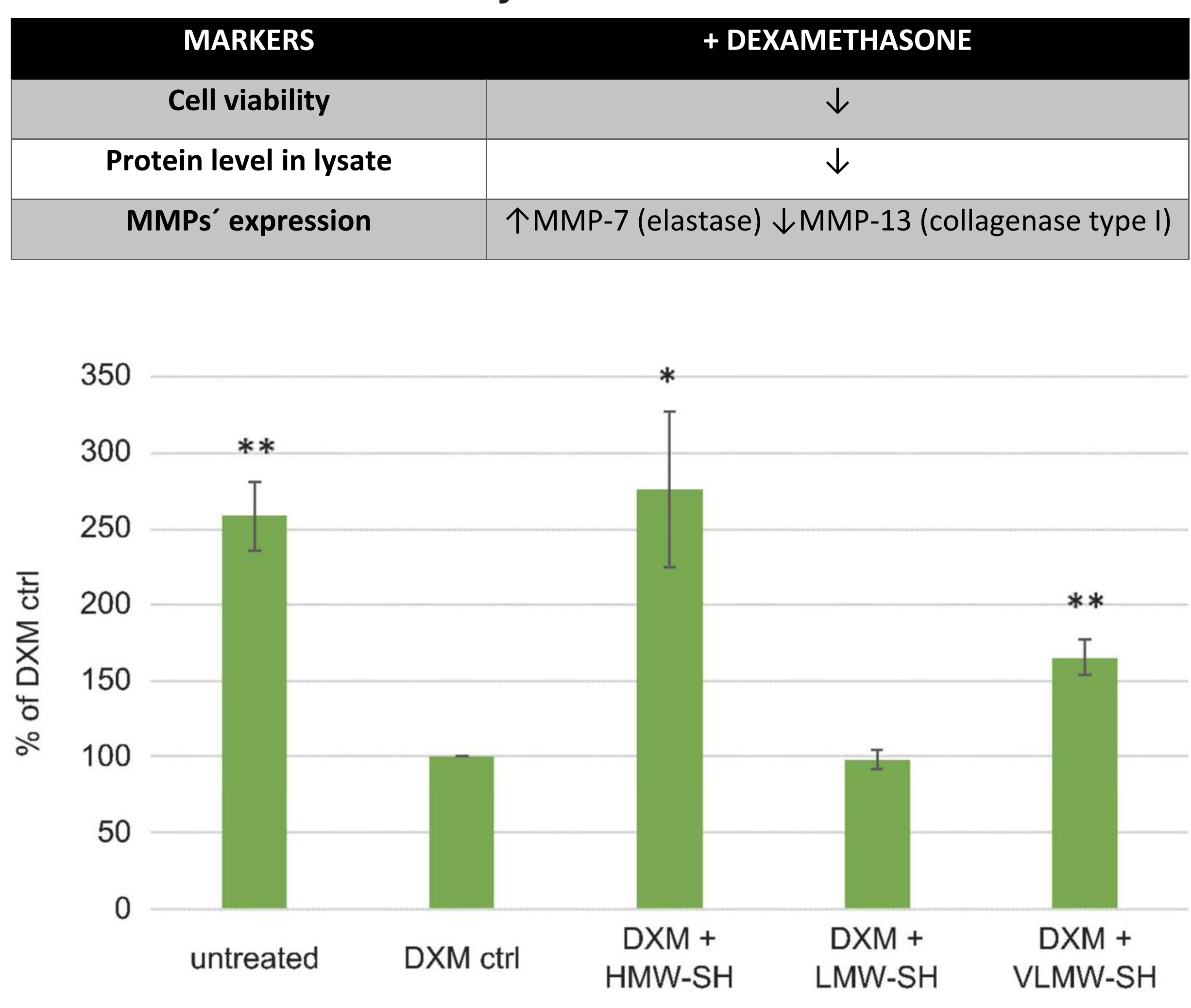

Figure 2. The viability of atrophic $\mathrm{HaCaT}$ keratinocytes $(+/-$ SH). Untreated = non-atrophic; DXM = atrophic. The viability was determined using resazurin assay. The values represent means + SEM of the of $\mathrm{HaCaT}$ keratinocytes viability $(*$ P $0.05,{ }^{* *} p \leq 0.01,{ }^{* \star *} p \leq 0.001$ compared to DXM ctrl, $n=5$ ).

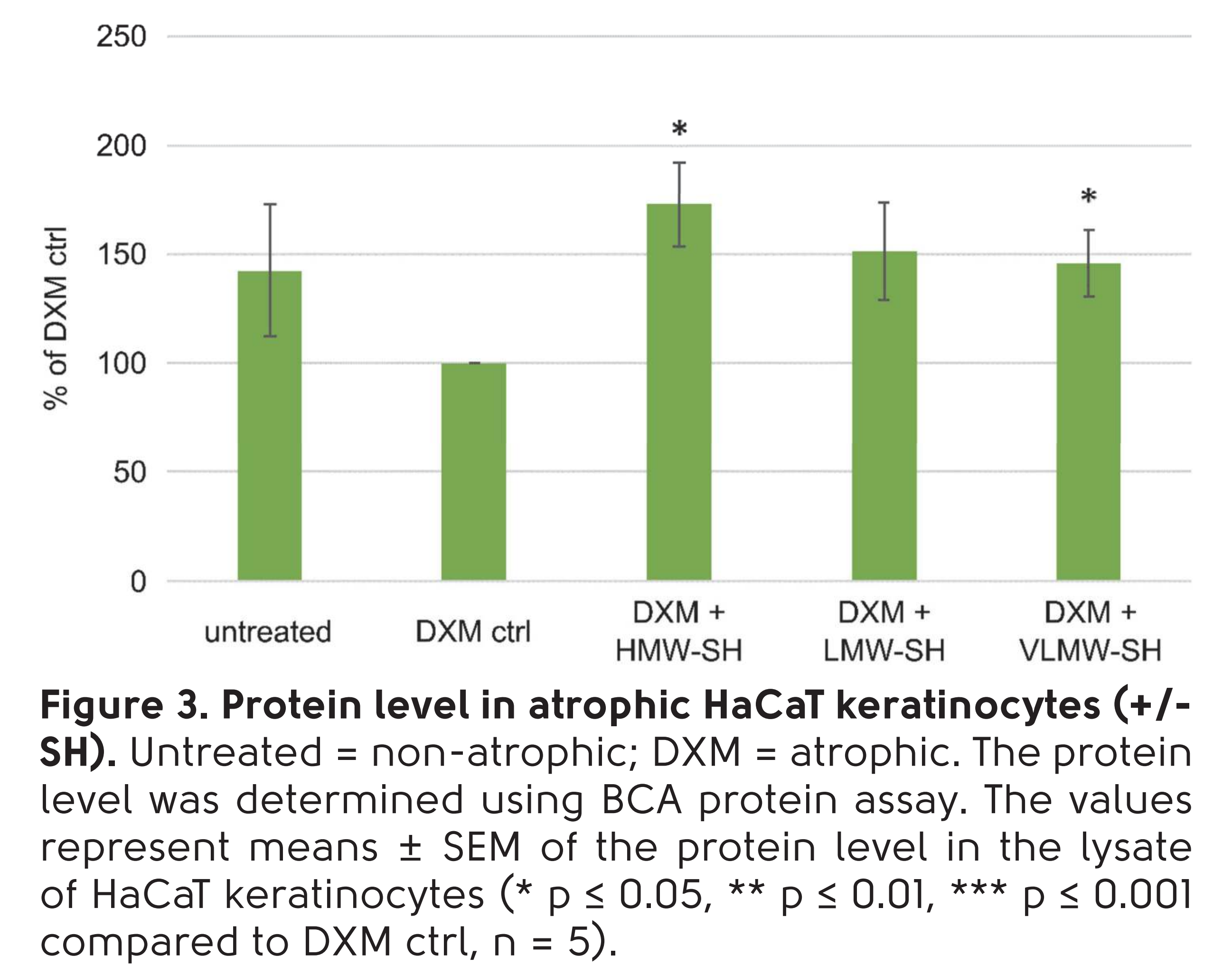

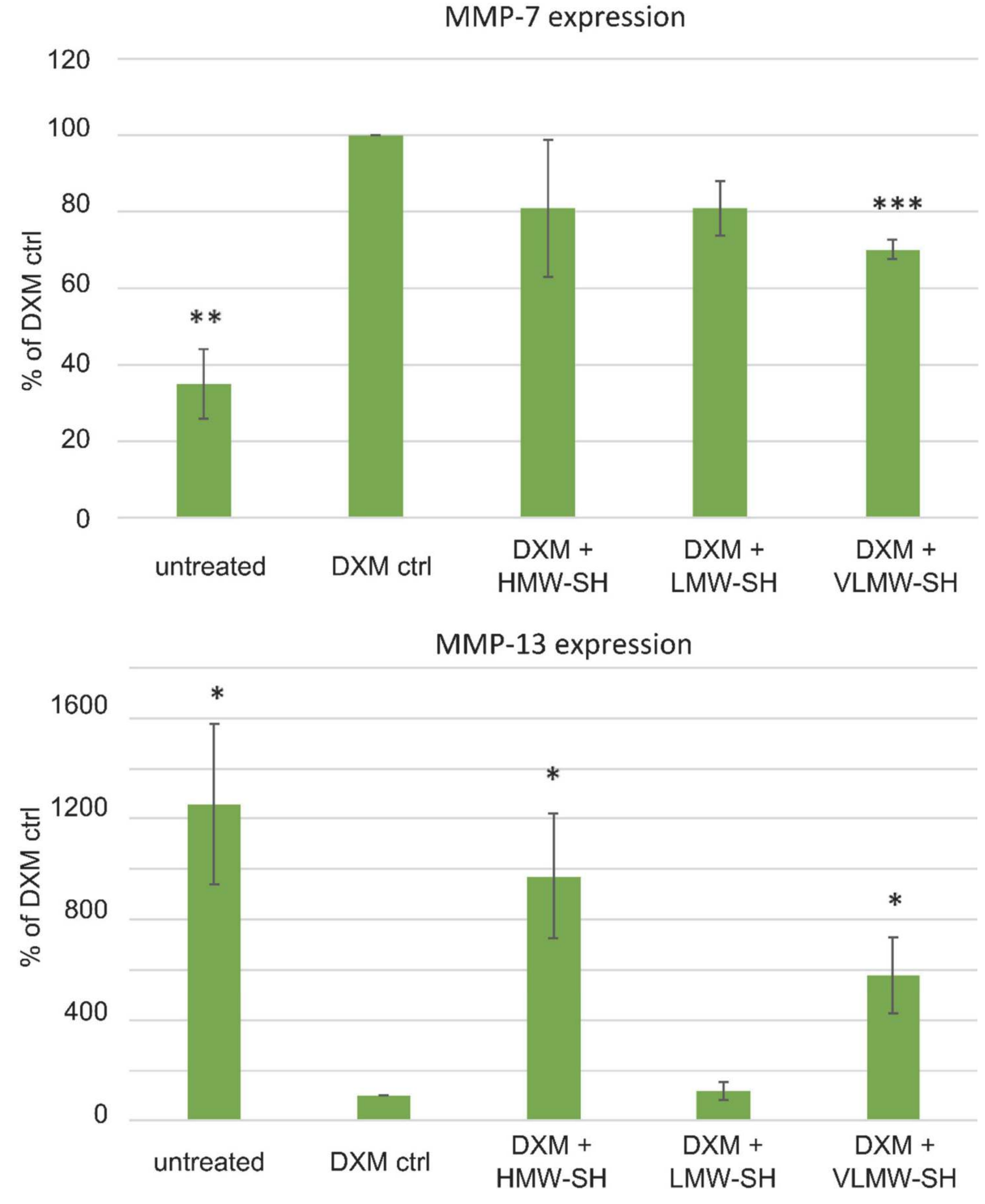

Figure 4. MMP-7 and MMP-13 gene expression level in atrophic HaCaT keratinocytes $(+/-\mathrm{SH})$. Untreated $=$ non-atrophic: DXM = atrophic. The gene expression was determined using real-time QRT-PCR. The values represent means + SEM of the gene expression of MMP-7/MMP-13 in HaCaT keratinocytes g* $P \leq 0.05^{* *} \mathrm{P} \leq 0.01^{* * *} \mathrm{P} \leq 0.001$ compared to DXM ctrl $(* x \leq 0.05, \quad p \leq 0.01, \quad p \leq 0.001$ compared to DXM ctrl,
$n=5)$. IN VIVOANTI-ATROPHICEFFECTOFVLMW SODIUM HYALURONATE

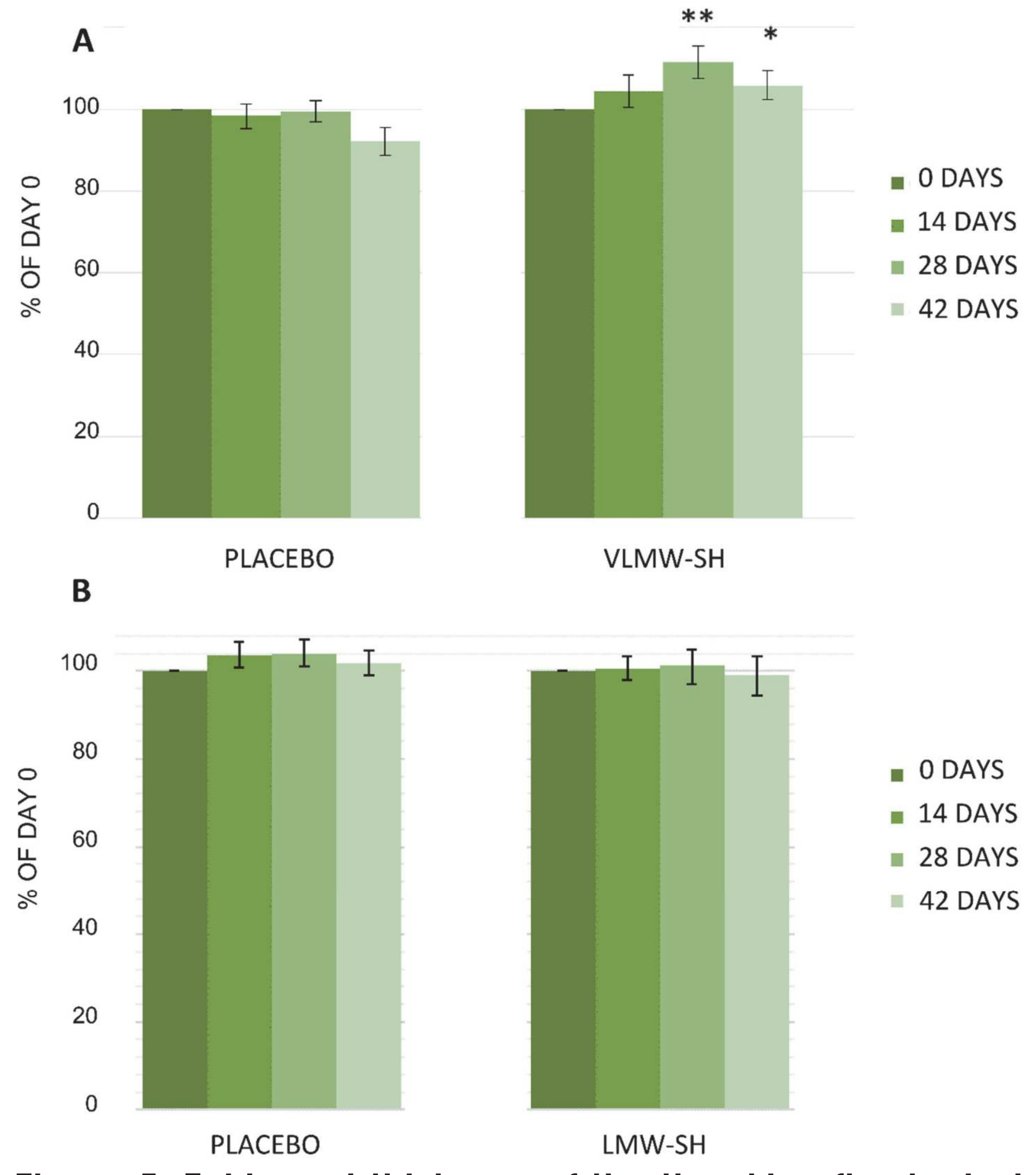

Figure 5. Epidermal thickness of the the skin after topical application of 0,2\% VLMW-SH (A) and 0,2\% LMW-SH (B). In contrast with LMW-SH, VLMW-SH significantly increased the thickness of living epidermis. It was determined using in vivo reflectance confocal microscopy. In both (A) and (B) double-blind, split-face and placebo-controlled studies, 28 healthy female volunteers with living epidermal thickness below $50 \mu \mathrm{m}$ participated. The values represent means \pm SEM of the epidermal thickness (* $P \leq 0.05, * * p \leq 0.01, * \star * ~ P$ $\leq 0.001$ compared to placebo, $n=28$ ).

\section{REFERENCES}

Kaya, G. et al. Hyaluronate Fragments Reverse Skin Atrophy by a CD44-Dependent Mechanism. PLOS Med. 3 e493 (2006) 